\title{
Cross-reactivity and inclusivity analysis of CRISPR-based diagnostic assays of coronavirus SARS-CoV-2
}

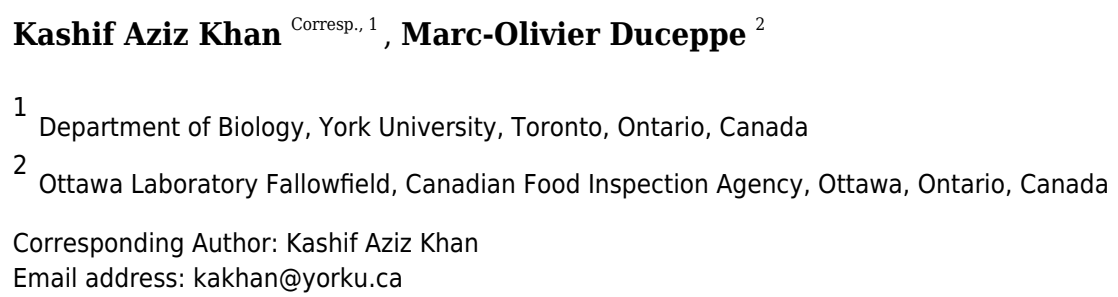

Severe acute respiratory syndrome coronavirus 2 (SARS-CoV-2; initially named as 2019$\mathrm{nCoV}$ ) is the cause of the novel coronavirus disease 2019 (COVID-19) pandemic. Its diagnosis relies on the molecular detection of the viral RNA by polymerase chain reaction (PCR) while newer rapid CRISPR-based diagnostic tools are being developed. As molecular diagnostic assays rely on the detection of unique sequences of viral nucleic acid, the target regions must be common to all coronavirus SARS-CoV-2 circulating strains, yet unique to SARS-CoV-2 with no cross-reactivity with the genome of the host and other normal or pathogenic organisms potentially present in the patient samples. This stage 1 protocol proposes in silico cross-reactivity and inclusivity analysis of the recently developed CRISPR-based diagnostic assays. Cross-reactivity will be analyzed through comparison of target regions with the genome sequence of the human, 7 coronaviruses and 21 other organisms. Inclusivity analysis will be performed through the verification of the sequence variability within the target regions using publicly available SARS-CoV-2 sequences from around the world. The absence of cross-reactivity and any mutations in target regions of the assay used would provide a higher degree of confidence in the CRISPR-based diagnostic tests being developed while the presence could help guide the assay development efforts. We believe that this study would provide potentially important information for clinicians, researchers, and decision-makers. 
1 Cross-reactivity and inclusivity analysis of CRISPR-

2 based diagnostic assays of coronavirus SARS-CoV-2

3

4 Kashif Aziz Khan ${ }^{1}$ and Marc-Olivier Duceppe ${ }^{2}$

$5 \quad{ }^{1}$ Department of Biology, York University, Toronto, ON, Canada.

$6{ }^{2}$ Ottawa Laboratory Fallowfield, Canadian Food Inspection Agency, Ottawa, ON, Canada.

7

8 Corresponding Author:

9 Kashif Aziz Khan

10 Email address: kakhan@yorku.ca 


\section{Abstract:}

Severe acute respiratory syndrome coronavirus 2 (SARS-CoV-2; initially named as 2019-nCoV) is the cause of the novel coronavirus disease 2019 (COVID-19) pandemic. Its diagnosis relies on the molecular detection of the viral RNA by polymerase chain reaction (PCR) while newer rapid CRISPR-based diagnostic tools are being developed. As molecular diagnostic assays rely on the detection of unique sequences of viral nucleic acid, the target regions must be common to all coronavirus SARS-CoV-2 circulating strains, yet unique to SARS-CoV-2 with no crossreactivity with the genome of the host and other normal or pathogenic organisms potentially present in the patient samples. This stage 1 protocol proposes in silico cross-reactivity and inclusivity analysis of the recently developed CRISPR-based diagnostic assays. Cross-reactivity will be analyzed through comparison of target regions with the genome sequence of the human, 7 coronaviruses and 21 other organisms. Inclusivity analysis will be performed through the verification of the sequence variability within the target regions using publicly available SARSCoV-2 sequences from around the world. The absence of cross-reactivity and any mutations in target regions of the assay used would provide a higher degree of confidence in the CRISPRbased diagnostic tests being developed while the presence could help guide the assay development efforts. We believe that this study would provide potentially important information for clinicians, researchers, and decision-makers.

\section{Introduction:}

Severe acute respiratory syndrome coronavirus 2 (SARS-CoV-2; initially named as 2019-nCoV) was firstly isolated from a cluster of pneumonia patients in Wuhan, China and is the cause of novel coronavirus disease termed COVID-19 (Wu et al. 2020; Zhou et al. 2020; Zhu et al. 2020). The rapid spread of the virus has resulted in a declaration of a global pandemic by the World Health Organization (WHO) reaching more than 220 countries and territories (WHO 2020c). SARS-CoV-2 has been classified as a member of the family Coronaviridae in the genus

Betacoronavirus along with SARS-CoV and the Middle East respiratory syndrome (MERS)$38 \mathrm{CoV}$ (Gorbalenya et al. 2020). The sequencing of the virus from patients early in the outbreak has shown that its single-stranded RNA genome is $\sim 30 \mathrm{~kb}$ in size (Chan et al. 2020; Lu et al. 
2020; Wu et al. 2020). The SARS-CoV-2 genome has been predicted to encode at least 10 open reading frames (ORFs) for structural and accessory proteins, based on similarity with SARSCoV. As per current annotation (NC_045512.2), these viral ORFs encode replicase ORF1ab, spike (S), envelope (E), membrane (M), nucleocapsid $(\mathrm{N})$, and at least 6 accessory proteins (3a, 6, 7a, 7b, 8, and 10) (NCBI 2020). The pandemic has serious public health and economic implications. The day-to-day life of billions of people has been affected due to different forms of social distancing measures in place in different parts of the world to mitigate the spread of the virus. Thus, the widespread availability of rapid and reliable diagnostic testing is an important tool for policymakers to make public health decisions. The current diagnosis of COVID-19 relies on the molecular detection of viral RNA from patient samples using nucleic acid amplification tests (NAAT) like polymerase chain reaction (PCR) (WHO 2020b). However, PCR requires specialized equipment and trained staff to perform the test and interpret the results, and thus is a challenge for remote low-resource settings. One of the alternatives being explored is the CRISPR-based nucleic-acid detection methods that may be particularly useful for screening outside the laboratory setting, for example at point-of-care, airports, offices and homes.

CRISPR-Cas (디 ustered regularly interspaced short palindromic repeats-CRISPR-associated), a component of the bacterial immune system to infectious nucleic acid, has been widely used as a gene-editing tool. This technology exploits the ability of Cas proteins to accurately target any region in DNA in association with CRISPR RNA (crRNA) that matches the target DNA with or without the requirement of a protospacer adjacent motif (PAM) (Moon et al. 2019). Initially explored for Cas9 protein (Pardee et al. 2016), the application of CRISPR in nucleic acid detection emerged as a viable tool with the discovery of promiscuous collateral cleavage activity of Cas12a (formerly Cpf1), Cas12b (formerly C2c1) and Cas13a (formerly C2c2) after target recognition (Chen et al. 2018; Gootenberg et al. 2017). Several CRISPR-based methods have been developed for the detection of RNA and DNA viruses (Jia et al. 2020; Strich et al. 2019). With the emergence of the novel coronavirus, scientists are rapidly employing these tools for the detection of SARS-CoV-2 from patient samples as an alternative to PCR. The Cas12a has been used for the diagnosis of COVID-19 from patient samples targeting viral genes $\mathrm{N}$ and $\mathrm{E}$ (Broughton et al. 2020). Similarly, Cas12b, Cas13a and FuCas9-based assays have also been developed for the detection of SARS-CoV-2 (Ackerman et al. 2020; Azhar et al. 2021; Guo et al. 2020). Cas12a-based DETECTR and Cas13a-based SHERLOCK have been approved by the US 
71 Food and Drug Administration (FDA) under Emergency Use Authorization (Mammoth 2020;

72 Sherlock 2020) and FuCas9-based FELUDA has been approved for diagnosis of COVID-19 in

73

74

75 India (Mitra 2020). Several other CRISPR-based methods are also under development (Petrillo et al. 2020).

The molecular diagnosis of SARS-CoV-2 may be jeopardized by potential preanalytical and analytical vulnerabilities leading to false-positive or false-negative results (Lippi et al. 2020). As molecular diagnostic assays rely on the detection of unique sequences of viral nucleic acid, these are prone to mismatches due to genetic variability in the viral genome as well as cross-reactivity with the nucleic acid of other organisms present in the samples. The selectivity of an assay is generally validated in a laboratory using target strains, near-neighbour strains and other organisms. The use of bioinformatics tools and genome sequence databases can help to reduce wet-lab testing to a narrower focus and help to estimate more accurately the false-positive and false-negative rates of an assay (SantaLucia et al. 2020). It is known that mutations at primer/probe binding regions of the viral genome can result in potential mismatches and falsenegative PCR diagnoses (Lefever et al. 2013; Stadhouders et al. 2010; Whiley \& Sloots 2005). We and others have concurrently demonstrated the genetic variability in the primer/probe binding regions of the SARS-CoV-2 genome highlighting the importance of periodic sequence verification for optimal virus detection (Farkas et al. 2020; Khan \& Cheung 2020; Osorio \& Correia-Neves 2020). Assay specificity remains a focus area in CRISPR-diagnostics as Cas proteins can result in a false-positive diagnosis due to their intrinsic capacity of mismatch tolerance. This risk has been minimized with the newer Cas proteins, Cas12a, Cas12b and Cas13, that have a lower tolerance for mismatches compared to front-runner Cas9 especially in the "seed" region (Safari et al. 2019). However, this raises the possibility that these tests may miss certain viral variants due to genetic variability in the regions targeted by these assays. The mismatch intolerant seed region of $\sim 6$ nucleotides is located in the PAM-proximal region for Cas12a (Chen et al. 2018; Kim et al. 2016) while the seed region is located in the central region of crRNA for Cas13a (Abudayyeh et al. 2017; Cox et al. 2017). Francisella novicida Cas9 (FnCas9) has been reported to have higher specificity and lower tolerance for mismatches compared to Streptococcus pyogenes Cas9 ( $\mathrm{SpCas9)}$ tolerating only a single mismatch especially at the PAM-distal seed end (Acharya et al. 2019). It has been shown that even two mismatches would impede or even abolish the trans-cleavage activity of Alicyclobacillus acidiphilus Cas $12 \mathrm{~b}$ 
102 (AaCas12b/AapCas12b) (Teng et al. 2019). It is important to design crRNA adjacent to Cas-

103 specific PAM that is common to all SARS-CoV-2 strains, yet unique to SARS-CoV-2

104 coronavirus with no cross-reactivity with the genome of the host and other normal or pathogenic

105 organisms potentially present in the patient samples.

106 The objective of this study is the verification of the cross-reactivity and sequence variability

107 within the target regions of CRISPR-based COVID-19 diagnostic assays, using publicly

108 available sequence databases. The absence of any cross-reactivity and mutations in target regions

109 of the assay used would provide a higher degree of confidence in the alternative tests being

110 developed while the presence of mutations could help guide assay development efforts. We

111 believe that this study would provide important information for clinicians, researchers and 112 policy-makers.

\section{Methods:}

115 CRISPR-based diagnostic assays and SARS-CoV-2 sequences

116 At least 15 crRNA of recently published CRISPR-based methods will be selected based on the

117 literature review. The cross-reactivity and sequence variability within the target regions of

118 CRISPR-based diagnostic assays will be determined using the protocol described below. The

119 design planner is included in Table S1. The source code is available from the GitHub repository

120 (https://github.com/duceppemo/CRISPR_Assay_Tester) and is easily installable with the Conda

121 package manager. The script will be validated (and updated if necessary) as per the method

122 described earlier (Khan \& Cheung 2020).

123 SARS-CoV-2 genome sequences deposited by laboratories around the world will be obtained

124 from the NCBI virus database (Hatcher et al. 2017). A total of $400000^{*}$ near full-length

125 sequences will be downloaded by applying the complete filter. The RNA genome of SARS-CoV-

1262 is shown in DNA format as per scientific convention. The complete genome of Wuhan-Hu-1

127 isolate, which is $29,903 \mathrm{bp}$ long, will be included as a reference (NCBI accession number:

128 NC_045512.2). 
$129 *$ The number of sequences in the NCBI database is growing on a daily basis and the exact

130 number of included sequences would be updated in the $2^{\text {nd }}$ stage submission.

131 Cross-reactivity analysis:

132 Each crRNA sequence along with the PAM sequence, if applicable, (Table 1) will be analyzed

133 for reactivity with the genome sequence of the human, 7 coronaviruses and 21 other species

134 including normal or pathogenic organisms that may potentially be present in patient samples.

135 The complete list to be tested can be found in Table S2. This step will be performed using

136 GGGenome nucleotide sequence search online server (http://gggenome.dbcls.jp/) (Naito et al.

1372015 ) allowing several mismatches to check for $\geq 80 \%$ homology according to the requirement of

138 WHO's Emergency Use Listing for in vitro diagnostics detecting SARS-CoV-2 nucleic acid

139 (WHO 2020a). The potential hits on both orientations with different numbers of mismatches for

140 each crRNA will be returned along with a summary of number of hits for different organisms.

141 The hits with a match in the seed and the PAM region will be discussed.

142 Inclusivity Analysis:

143 Multiple sequence alignment (MSA) of SARS-CoV-2 sequences will be performed using

144 MAFFT (Multiple Alignment with Fast Fourier Transform) program v7.480 (Katoh \& Standley

145 2013) excluding low coverage sequences ( $>1 \%$ Ns) and using the Wuhan-Hu-1 genome

146 (NC_045512.2) as reference. To evaluate the sequence variability in the target regions of each

147 assay, referred here as the region of interest (ROI), the crRNA and PAM sequence will be

148 extracted from all the entries in the MAFFT alignment file using the coordinates determined

149 during cross-reactivity analysis. The MSA sequence for each ROI will be stratified by

150 segregating sequences into discrete groups of identical sequence variants along with their

151 frequency. To remove extremely low prevalent variants and sequencing errors in the data, only

152 the sequence variants occurring in more than $0.01 \%$ of all sequences will be further considered

153 by default. Sequences with ambiguous nucleotides and stretches of Ns in ROIs will be excluded

154 from the analysis. The summary of mutated nucleotides for each target region will be returned

155 and results will be reported as the frequency of hits with 100\% match, hits with mismatches, and

156 excluded sequences. The frequency of sequence variants with mismatches in the seed and PAM

157 region will be discussed. 
159

160

161

162

163

164

165

166

167

168

169

170

171

172

173

174

175

176

177

178

179

180

181

182

183

184

185

186

187

188

189

190

191

192

193

194

195

196

197

198

199

200

201

202

203

\section{References}

Abudayyeh OO, Gootenberg JS, Essletzbichler P, Han S, Joung J, Belanto JJ, Verdine V, Cox DBT, Kellner MJ, Regev A, Lander ES, Voytas DF, Ting AY, and Zhang F. 2017. RNA targeting with CRISPR-Cas13. Nature 550:280-284. 10.1038/nature24049

Acharya S, Mishra A, Paul D, Ansari AH, Azhar M, Kumar M, Rauthan R, Sharma N, Aich M, Sinha D, Sharma S, Jain S, Ray A, Jain S, Ramalingam S, Maiti S, and Chakraborty D. 2019. Francisella novicida Cas9 interrogates genomic DNA with very high specificity and can be used for mammalian genome editing. Proceedings of the National Academy of Sciences 116:20959-20968. 10.1073/pnas.1818461116

Ackerman CM, Myhrvold C, Thakku SG, Freije CA, Metsky HC, Yang DK, Ye SH, Boehm CK, Kosoko-Thoroddsen TF, Kehe J, Nguyen TG, Carter A, Kulesa A, Barnes JR, Dugan VG, Hung DT, Blainey PC, and Sabeti PC. 2020. Massively multiplexed nucleic acid detection with Cas13. Nature 582:277-282. 10.1038/s41586-020-2279-8

Azhar M, Phutela R, Kumar M, Ansari AH, Rauthan R, Gulati S, Sharma N, Sinha D, Sharma S, Singh S, Acharya S, Sarkar S, Paul D, Kathpalia P, Aich M, Sehgal P, Ranjan G, Bhoyar RC, Indian Co VG, Genetic Epidemiology C, Singhal K, Lad H, Patra PK, Makharia G, Chandak GR, Pesala B, Chakraborty D, and Maiti S. 2021. Rapid and accurate nucleobase detection using FnCas9 and its application in COVID-19 diagnosis. Biosens Bioelectron 183:113207. 10.1016/j.bios.2021.113207

Broughton JP, Deng X, Yu G, Fasching CL, Servellita V, Singh J, Miao X, Streithorst JA, Granados A, Sotomayor-Gonzalez A, Zorn K, Gopez A, Hsu E, Gu W, Miller S, Pan CY, Guevara H, Wadford DA, Chen JS, and Chiu CY. 2020. CRISPR-Cas12-based detection of SARS-CoV-2. Nature Biotechnology. 10.1038/s41587-020-0513-4

Chan JF, Kok KH, Zhu Z, Chu H, To KK, Yuan S, and Yuen KY. 2020. Genomic characterization of the 2019 novel human-pathogenic coronavirus isolated from a patient with atypical pneumonia after visiting Wuhan. Emerg Microbes Infect 9:221-236. 10.1080/22221751.2020.1719902

Chen JS, Ma E, Harrington LB, Da Costa M, Tian X, Palefsky JM, and Doudna JA. 2018. CRISPR-Cas12a target binding unleashes indiscriminate single-stranded DNase activity. Science 360:436-439. 10.1126/science.aar6245

Cox DBT, Gootenberg JS, Abudayyeh OO, Franklin B, Kellner MJ, Joung J, and Zhang F. 2017. RNA editing with CRISPR-Cas13. Science 358:1019-1027. 10.1126/science.aaq0180

Farkas C, Fuentes-Villalobos F, Garrido JL, Haigh J, and Barría MI. 2020. Insights on early mutational events in SARS-CoV-2 virus reveal founder effects across geographical regions. PeerJ 8. 10.7717/peerj.9255

Gootenberg JS, Abudayyeh OO, Lee JW, Essletzbichler P, Dy AJ, Joung J, Verdine V, Donghia N, Daringer NM, Freije CA, Myhrvold C, Bhattacharyya RP, Livny J, Regev A, Koonin EV, Hung DT, Sabeti PC, Collins JJ, and Zhang F. 2017. Nucleic acid detection with CRISPR-Cas13a/C2c2. Science 356:438-442. 10.1126/science.aam9321

Gorbalenya AE, Baker SC, Baric RS, Groot RJd, Drosten C, Gulyaeva AA, Haagmans BL, Lauber C, Leontovich AM, Neuman BW, Penzar D, Perlman S, Poon LLM, Samborskiy DV, Sidorov IA, Sola I, Ziebuhr J, and Coronaviridae Study Group of the International Committee on Taxonomy of V. 2020. The species Severe acute respiratory syndromerelated coronavirus: classifying 2019-nCoV and naming it SARS-CoV-2. Nat Microbiol 5:536-544. 10.1038/s41564-020-0695-z 
204

205

206

207

208

209

210

211

212

213

214

215

216

217

218

219

220

221

222

223

224

225

226

227

228

229

230

231

232

233

234

235

236

237

238

239

240

241

242

243

244

245

246

247

248

Guo L, Sun X, Wang X, Liang C, Jiang H, Gao Q, Dai M, Qu B, Fang S, Mao Y, Chen Y, Feng G, Gu Q, Wang RR, Zhou Q, and Li W. 2020. SARS-CoV-2 detection with CRISPR diagnostics. Cell Discovery 6. 10.1038/s41421-020-0174-y

Hatcher EL, Zhdanov SA, Bao Y, Blinkova O, Nawrocki EP, Ostapchuck Y, Schaffer AA, and Brister JR. 2017. Virus Variation Resource - improved response to emergent viral outbreaks. Nucleic Acids Res 45:D482-D490. 10.1093/nar/gkw1065

Jia F, Li X, Zhang C, and Tang X. 2020. The expanded development and application of CRISPR system for sensitive nucleotide detection. Protein \& Cell. 10.1007/s13238-020-00708-8

Katoh K, and Standley DM. 2013. MAFFT Multiple Sequence Alignment Software Version 7: Improvements in Performance and Usability. Molecular Biology and Evolution 30:772780. $10.1093 / \mathrm{molbev} / \mathrm{mst} 010$

Khan KA, and Cheung P. 2020. Presence of mismatches between diagnostic PCR assays and coronavirus SARS-CoV-2 genome. Royal Society Open Science 7:200636. doi:10.1098/rsos.200636

Kim D, Kim J, Hur JK, Been KW, Yoon S-h, and Kim J-S. 2016. Genome-wide analysis reveals specificities of Cpfl endonucleases in human cells. Nature Biotechnology 34:863-868. 10.1038/nbt.3609

Lefever S, Pattyn F, Hellemans J, and Vandesompele J. 2013. Single-nucleotide polymorphisms and other mismatches reduce performance of quantitative PCR assays. Clin Chem 59:1470-1480. 10.1373/clinchem.2013.203653

Lippi G, Simundic AM, and Plebani M. 2020. Potential preanalytical and analytical vulnerabilities in the laboratory diagnosis of coronavirus disease 2019 (COVID-19). Clin Chem Lab Med. 10.1515/cclm-2020-0285

Lu R, Zhao X, Li J, Niu P, Yang B, Wu H, Wang W, Song H, Huang B, Zhu N, Bi Y, Ma X, Zhan F, Wang L, Hu T, Zhou H, Hu Z, Zhou W, Zhao L, Chen J, Meng Y, Wang J, Lin Y, Yuan J, Xie Z, Ma J, Liu WJ, Wang D, Xu W, Holmes EC, Gao GF, Wu G, Chen W, Shi W, and Tan W. 2020. Genomic characterisation and epidemiology of 2019 novel coronavirus: implications for virus origins and receptor binding. Lancet 395:565-574. 10.1016/S0140-6736(20)30251-8

Mammoth. 2020. SARS-CoV-2 DETECTR Reagent Kit. Available at https://www.fda.gov/media/141765/download (accessed Septermber 3 2020).

Mitra E. 2020. India's drug authority approved paper-strip Covid-19 test that could return results within hour. CNN.

Moon SB, Kim DY, Ko J-H, and Kim Y-S. 2019. Recent advances in the CRISPR genome editing tool set. Experimental \& Molecular Medicine 51:1-11. 10.1038/s12276-0190339-7

Naito Y, Hino K, Bono H, and Ui-Tei K. 2015. CRISPRdirect: software for designing CRISPR/Cas guide RNA with reduced off-target sites. Bioinformatics 31:1120-1123. 10.1093/bioinformatics/btu743

NCBI. 2020. Sequence Viewer 3.36.0: Severe acute respiratory syndrome coronavirus 2 isolate Wuhan-Hu-1, complete genome. Available at https://www.ncbi.nlm.nih.gov/projects/sviewer/?id=NC 045512.2 (accessed May 15 2020).

Osorio NS, and Correia-Neves M. 2020. Implication of SARS-CoV-2 evolution in the sensitivity of RT-qPCR diagnostic assays. Lancet Infect Dis. 10.1016/S1473-3099(20)30435-7 
249

250

251

252

253

254

255

256

257

258

259

260

261

262

263

264

265

266

267

268

269

270

271

272

273

274

275

276

277

278

279

280

281

282

283

284

285

286

287

288

289

290

291

292

293

Pardee K, Green AA, Takahashi MK, Braff D, Lambert G, Lee JW, Ferrante T, Ma D, Donghia N, Fan M, Daringer NM, Bosch I, Dudley DM, O'Connor DH, Gehrke L, and Collins JJ. 2016. Rapid, low-cost detection of Zika virus using programmable biomolecular components. Cell 165:1255-1266. 10.1016/j.cell.2016.04.059

Petrillo M, Querci M, Tkachenko O, Siska IR, Ben E, Angers-Loustau A, Bogni A, Brunetto A, Fabbri M, Garlant L, Lievens A, Munoz A, Paracchini V, Pietretti D, Puertas-Gallardo A, Raffael B, Sarno E, Tregoat V, Zaro F, and Van den Eede G. 2020. The EU one-stopshop collection of publicly available information on COVID-19 in vitro diagnostic medical devices. F1000Res 9:1296. 10.12688/f1000research.27308.1

Safari F, Zare K, Negahdaripour M, Barekati-Mowahed M, and Ghasemi Y. 2019. CRISPR Cpf1 proteins: structure, function and implications for genome editing. Cell \& Bioscience 9 . 10.1186/s13578-019-0298-7

SantaLucia J, Sozhamannan S, Gans JD, Koehler JW, Soong R, Lin NJ, Xie G, Olson V, Roth K, and Beck L. 2020. Appendix Q: Recommendations for developing molecular assays for microbial pathogen detection using modern in silico approaches. J AOAC Int 103:882899. 10.1093/jaoacint/qsaa045

Sherlock. 2020. Sherlock CRISPR SARS-CoV-2 kit. Available at https://www.fda.gov/media/137746/download (accessed July 32020 ).

Stadhouders R, Pas SD, Anber J, Voermans J, Mes TH, and Schutten M. 2010. The effect of primer-template mismatches on the detection and quantification of nucleic acids using the 5' nuclease assay. J Mol Diagn 12:109-117. 10.2353/jmoldx.2010.090035

Strich JR, Chertow DS, and Kraft CS. 2019. CRISPR-Cas biology and its application to infectious diseases. Journal of Clinical Microbiology 57. 10.1128/jcm.01307-18

Teng F, Guo L, Cui T, Wang X-G, Xu K, Gao Q, Zhou Q, and Li W. 2019. CDetection: CRISPR-Cas12b-based DNA detection with sub-attomolar sensitivity and single-base specificity. Genome Biology 20. 10.1186/s13059-019-1742-z

Whiley DM, and Sloots TP. 2005. Sequence variation in primer targets affects the accuracy of viral quantitative PCR. J Clin Virol 34:104-107. 10.1016/j.jcv.2005.02.010

WHO. 2020a. Instructions and requirements for Emergency Use Listing (EUL) submission: In vitro diagnostics detecting SARS-CoV-2 nucleic acid and rapid diagnostics tests detecting SARS-CoV-2 antigens. Available at https://www.who.int/publications/m/item/PQDx-347-version-4 (accessed December 31 2020).

WHO. 2020b. Laboratory testing for coronavirus disease (COVID-19) in suspected human cases: Interim Guidance. Available at https://www.who.int/publications/i/item/10665-331501 (accessed July 32020 ).

WHO. 2020c. WHO Coronavirus Disease (COVID-19) Dashboard. Available at https://covid19. who.int/ (accessed December 31 2020).

Wu F, Zhao S, Yu B, Chen YM, Wang W, Song ZG, Hu Y, Tao ZW, Tian JH, Pei YY, Yuan ML, Zhang YL, Dai FH, Liu Y, Wang QM, Zheng JJ, Xu L, Holmes EC, and Zhang YZ. 2020. A new coronavirus associated with human respiratory disease in China. Nature 579:265-269. 10.1038/s41586-020-2008-3

Zhou P, Yang XL, Wang XG, Hu B, Zhang L, Zhang W, Si HR, Zhu Y, Li B, Huang CL, Chen HD, Chen J, Luo Y, Guo H, Jiang RD, Liu MQ, Chen Y, Shen XR, Wang X, Zheng XS, Zhao K, Chen QJ, Deng F, Liu LL, Yan B, Zhan FX, Wang YY, Xiao GF, and Shi ZL.

Peer) reviewing PDF | (2020:07:50961:1:1:NEW 24 Jul 2021) 
294 2020. A pneumonia outbreak associated with a new coronavirus of probable bat origin. $295 \quad$ Nature 579:270-273. 10.1038/s41586-020-2012-7

296 Zhu N, Zhang D, Wang W, Li X, Yang B, Song J, Zhao X, Huang B, Shi W, Lu R, Niu P, Zhan

297

298 F, Ma X, Wang D, Xu W, Wu G, Gao GF, Tan W, China Novel Coronavirus I, and Research T. 2020. A novel coronavirus from patients with pneumonia in China, 2019. N Engl J Med 382:727-733. 10.1056/NEJMoa2001017

300 
Table $\mathbf{1}$ (on next page)

Cas proteins used in CRISPR diagnostics 


\begin{tabular}{|l|l|l|l|}
\hline Cas variant & Enzyme & Organism & PAM \\
\hline Cas12 & $\begin{array}{l}\text { LbCas12a (or } \\
\text { LbaCas12a) }\end{array}$ & $\begin{array}{l}\text { Lachnospiraceae } \\
\text { bacterium }\end{array}$ & 5'-TTTN \\
\hline & $\begin{array}{l}\text { AaCas12b (or } \\
\text { AapCas12b) }\end{array}$ & $\begin{array}{l}\text { Alicyclobacillus } \\
\text { acidiphilus }\end{array}$ & 5'-TTN \\
\hline Cas13 & LbuCas13a & Leptotrichia buccalis & Not applicable \\
\hline & LwaCas13a/ & Leptotrichia wadei & Not applicable \\
\hline Cas9 & FnCas9 & Francisella novicida & NGG- 3' \\
\hline
\end{tabular}

1 